\title{
Underwater Sonar Systems: A Methodology to Generate Bathymetry and Sub-strata Data
}

\author{
Milind Naik \\ Research Vessel Management, \\ CSIR-National Institute of Oceanography, \\ Dona-Paula, Goa, India
}

\author{
Govind Ranade \\ Marine Geo-Instrumentation, \\ CSIR-National Institute of Oceanography, \\ Dona-Paula, Goa, India
}

\author{
Rajesh B. Lohani \\ Department of E \& T C., \\ Goa College of Engineering, \\ Farmagudi, Ponda, Goa, India
}

\begin{abstract}
Data collection for logical interpretation of the observations made in any branch of science is solely dependent on the method or an approach to a particular problem. The techniques therefore followed to obtain complete or near complete solution depend upon the type of instrument used to make these observations. A morphological observation method to understand the sea floor involves a complex set of data collection to arrive at proper interpretation of a morphological feature. In the marine environment, careful system design is essential to overcome errors due to unstable nature of observation platform and the medium, which undergoes a continuous process of random or periodic variations.
\end{abstract}

The ultimate goal of a bathymetric survey is to produce a bathymetric map of an area surveyed with a certain geographical reference frame.[1] The other important aspect of bathymetric observations is for geological and geophysical exploratory type of surveys, which provide a vital link between studying morphological variations of the sea-floor and their impact on drawing inference on tectonic changes as a primary evidence of the earth-ocean dynamics. This data can provide a great insight in earth evolutionary processes on continental shelf dynamics, canyon and sea-mount formations, riverine fans extending to large distances into ocean basins. Bathymetry data obtained for any area is very useful in gravity anomaly studies etc. In all these observations, a good navigation system plays a key role of fixing a proper geographical reference frame. This will need a specific knowledge of the medium, underwater acoustics and a number of complementary sensor data such as attitude and heave measurement in addition to a number of instruments available for the depth determination.

A sub-bottom profiler basically provides details of the substrata layering of the ocean floor. During the movement of terrestrial material through rivers and flows meeting the sea, gets deposited in the nearshore region initially. This deposited material in the form sand, clay or silt OR in a combination, moves towards the deeper ocean floor due to wave interaction and bottom currents. As new deposits keep accumulating, layers of these deposit are formed. In many cases, organic matter also gets entrapped with this material. At certain conditions of pressure (due to sediment mass) and temperature, this organic matter converts into hydrocarbon deposits or gas-hydrate deposit. Therefore the study of the substrata below the sea-bottom provides in-depth knowledge of possible hydrocarbon deposits. These layers also provide history of bottom evolution. Also with these sediments foraminifera species which die, get deposited. These species provide vital information about the climate in the past. An altogether new branch of oceanography termed as ?paleo-oceanography? has evolved. Therefore the subbottom profilers are an important sonar tool in ocean exploration.

\section{General Terms}

bathymetry, Seabed morphology

\section{Keywords}

Sonar, acoustic, multibeam, Sub-bottom profiler, echosounder

\section{INTRODUCTION}

Data collection for logical interpretation of the observations made in any branch of science is solely dependent on the method or an approach to a particular problem. The techniques therefore followed to obtain complete or near complete solution depend upon the type of instrument used to make these observations. A morphological observation method to determine the depth involves a complex set of data collection to arrive at proper interpretation of a problem. The instrumentation therefore involves a highly sophisticated assembly of different measurement sequences. In the marine environment, it becomes necessary to carefully design an equipment to overcome errors due to unstable nature of observation platform and the medium, which under goes a continuous process of random or periodic variations.

In last four decades a substantial improvement in the signal processing, data handling and computing facilities has completely changed design concept of bathymetric systems and have almost replaced the single-beam echo sounding process with a range of multibeam swath bathymetry systems. Unlike single narrow beam echo sounders, these are comprised of multi-narrow beam swath systems or interferometric side scan sonars, in which received signal phase measurement to determine arrival angles and slant range data can be converted into depth data and therefore into a bathymetric map. Several such systems were designed initially with a limited scope of coverage and were essentially useful in deep water operations due to beam geometry design parameters of transmit and receive signals. In this paper, multibeam sonar concept is introduced and compared with various available 
systems for operation in both shallow and deep-water applications. Different design parameters and modes of presentation of data collected using such systems are presented. The swath width of these systems ranges from 0.7 times the water depths of earlier systems to more than 7 times the water depths of the recent systems.

\section{DIFFERENT SYSTEMS AND THEIR APPLICATION}

The early history of depth measurements goes to lead line measurements. Sound is often used as a main source for the observations and measurement in the marine environment, mainly because the electromagnetic waves are strongly attenuated by the water. As such, all media can propagate sound to a greater or lesser extent. The propagation takes place because of the vibrating particles, which act on the particles in its neighbourhood and form a chain of vibrating particles. Theoretically, the sound can exist in an infinite band of frequencies, i.e. $0 \mathrm{~Hz}<f<\infty \mathrm{Hz}$. The frequencies in the useful sonar band in marine environment lie between $8 \mathrm{~Hz}$ to $1 \mathrm{MHz}$. The frequency band of the interest for the bathymetric exploration is divided into further groups depending on the requirements.

They can be grouped depending on their frequency spectrum as shown in Table 1

Table 1. Equipments and their frequency band

\begin{tabular}{|c|c|c|}
\hline S.No & FREQUENCY BAND & TYPE OF EQUIPMENT \\
\hline 1 & $10 \mathrm{kHz}$ to $500 \mathrm{kHz}$ & Echo sounders Multibeam Sonar Side Scan Sonar \\
\hline 2 & $40 \mathrm{~Hz}$ to $1200 \mathrm{~Hz}$ & Sparker Systems \\
\hline 3 & $500 \mathrm{~Hz}$ to $10 \mathrm{kHz}$ & Boomer Systems \\
\hline 4 & $8 \mathrm{~Hz}$ to 64 to $256 \mathrm{~Hz}$ & Deep Water high energy source like Airgun \\
\hline 5 & $500 \mathrm{~Hz}$ to $15 \mathrm{kHz}$. & Chirg Sonar \\
\hline 6 & 4 to $7 \mathrm{kHz}$ & Sub-Bottom Profilers \\
\hline
\end{tabular}

\subsection{Echosounder}

Echosounder of earlier days, due to constraints of techniques available in transducer designs and the then available electronics, were of large physical size and less accurate and broad beamed systems (roughly $30^{\circ}$ to $60^{\circ}$ ). These systems were not able to provide high-resolution depth measurements and in turn could produce only large-scale relief identification of the seafloor. A variety of echosounder with different frequencies have been used based on the mission requirements. The frequency and the beam width parameters play a major role in the resolution of a bathymetry data, apart from other parameters like pulse width. The choice of frequency has its own advantages and disadvantages in terms of attenuation, range resolution and angular resolution. High frequency suffers from attenuation problem. However, the advantages are smaller transducer size, comparatively narrow beam width and ability to resolve smaller objects due to smaller wavelengths of high frequency signals. If used on a towed or any kind of submersible platform (AUV, ROV), the high frequency systems do not have near field operational constraints and can be operated close to bottom. The system will also have better bandwidth at higher frequencies and provide therefore a better range resolution. Due to higher attenuation of high frequencies, the water-bottom interface will provide a sharp, unambiguous echo, which will not be possible with low frequency system that will incorporate merging of echoes from sub-bottom. In shallow waters with high frequency based echosounder, the range resolution achieved through a broad bandwidth will be in range of few millimetres to centimetres depending on the frequency of operation. However, due to poor angular resolution owing to comparatively broad beam angles and an incomplete coverage it may degrade to some extent. The range accuracy also depends on knowledge of the sound velocity profile in the water column, which incorporates ray-bending effects due thermocline structure of the water column. This effect is more pronounced in the upper water columns up to 1000 meters of water depths. Angular resolution is the ability to discriminate in an angular direction for depression and azimuth of the receiver transducer location with respect to echo source. A better-directed beam can be achieved by using a multi element transducer in an array form (linear or circular). This enables proper focusing of the acoustic energy in a required direction. This will ensure a better angular resolution. Range resolution is an ability to discriminate in time (in case of TWTT calculations), which is dependent on the radial distance from the source. Range resolution as mentioned above is directly related to bandwidth of the transmit pulse.

In a basic bathymetry system or for that matter in any underwater acoustic instrumentation it becomes necessary to understand basic fundamentals regarding the underwater acoustics and the various sensors that make a bathymetry system. All the bathymetric or underwater acoustic systems are governed by the sonar equation that generally defines the echo signal detection as Echo Excess (EE) as follows: [2], [3]

$$
E E=S L-2 T L-(N L-D I)+B S-D T
$$

where

$\mathrm{SL}=$ source level

$\mathrm{TL}=$ transmission loss,

$\mathrm{NL}=$ noise Level,

$\mathrm{DI}=$ directivity index,

$\mathrm{BS}=$ bottom backscattering strength and

DT $=$ detection threshold .

Source level (SL) provides information on the signal intensity level to a referred to a one-meter distance from the centre of the source of a referred plane wave pressure of $1 \mathrm{mPa}$. The noise level (NL) is the environmental dependent noise spectrum of the medium and is dependent on the transducer frequency bandwidth. Directivity index (DI) is provided by the log value of the ratio of the intensity levels of the received energy and the reference energy level. The backscattering strength (BS) is dependent on the reflective nature of the sea bottom geometry. Finally the detection threshold (DT) is the system dependent parameter, which in general is a benchmark for the lowest level of the received signal, which can be considered as valid detectable signal.

\subsection{Multibeam Sonar}

A conventional echosounder can measure depths only immediately below the ship and for a satisfactory coverage of a survey area to produce a bathymetric chart it is required to plan many close grid parallel sounding survey lines. Even with a close grid survey it is possible to miss out some vital information about the topographic features, which may lie in-between two consecutive lines. Multibeam swath bathymetry systems came into existence, resulting in considerable reduction in the survey time and therefore became cost effective compared to normal single beam echo sounding application. The multibeam systems started with a swath coverage of up to $70 \%$ of the water depth and now there are systems available which can give a swath coverage of more than 
$300 \%$ of water depths in deep sea surveys going down to 10,000 meters of water depth, to about $750 \%$ of water depth for shallow to medium water depth surveys going to 1000 meters of water depth. This means that at a depth of 5000 meters, a system with $100 \%$ coverage capability can cover a cross section of 10 kilometres in one sweep giving about 60 to 100 depth points across the swath, compared to a single depth value with normal echosounder. The present day systems are capable of providing side scan imagery and backscatter information in addition to generating contour maps and $3 \mathrm{D}$ perspective of the surveyed area. The sonar mapping systems have provided the marine geophysicists, vital information on different geological structural formations like thrust faults, strike slip faults which occur due to parallel movement of the crustal fragments and are difficult to be identified using normal geophysical tools. It is important in a swath bathymetry system to have following parameters properly defined:

(1) Position (x, y, z),

(2) Orientation of transmit and receive beams $(\beta, \theta, \phi)$,

(3) Elevation (draft, heave), attitude monitoring (roll, pitch and now-a-days yaw motion),

(4) Refraction effects in Water column (i.e. ray bending), and

(5) Relative location of the sensor with respect to position.

The wide footprint transmitted by the MBES is segmented by the receiver array and related electronics into multiple smaller beams. The advantage of multi-beam over single beam echosounder as stated earlier is a large coverage of depth points in the ships direction during one ping. This provides $100 \%$ coverage of the bottom in an economic way. In deeper water the coverage becomes much wider compared to shallow depths. To define the above mentioned parameters in a swath bathymetry system the following ancillary system are used:[2],[4]

(1) Position (x, y, z): A good positioning system, which can deliver high accuracy position of the vessel. Some positioning system can deliver the RTK tide information, satellite corrections which give accuracy in terms of millimetres. The cost such system is high with higher accuracy and additional features.

(2) Orientation of transmit and receive beams $(\beta, \theta, \phi)$ : A sound velocity probe is mounted close to the transducer face, to orient the transmit and receive beams with reference to the changes in vessels attitude (roll, pitch, heave).

(3) Elevation (draft, heave), attitude monitoring: Motion sensor is installed close to CG of the vessel from where reference coordinate system for the installed sonar system is generated. This provides necessary corrections to compensate for change in the directions of transmit and received beams due to roll, pitch and heave of the vessel.

(4) Refraction effects in Water column: Due to density, temperature and salinity variations the transmitted and received signals undergo bending due to refraction owing to these changes. Therefore the measurement of sound velocity in the water column becomes necessary to apply these corrections to provide an accurate depth. This is achieved by using a sound velocity probe which traverses from the sea surface to desired survey depth.

(5) Relative location of the sensor with respect to position: At the time of installation of any sonar system, one needs to collocate other ancillary subsystems like hull mounted transducers, DGPS, Motion sensor in an appropriate ship coordinate system so that a reference frame is formed, with which all the measurements are carried out. For this, a dimensional survey of the vessel with all installed subsystem locations in a ship's coordinate system is carried out. This provides installation offset values resulting out of the installation process. These values are added in the installation parameters of the vessel, so that this forms a reference for computation of necessary correction to transmit and receive signals due to roll, pitch and heaving motion of the vessel.

\subsection{Sub-bottom Profiler}

Sub-bottom profiler is an acoustic system that is used to identify details and measure thicknesses of different sediment layers beneath the seabed. The sub-bottom profiler uses similar technique to that used by single-beam echo sounders, but uses lower frequencies, to achieve higher penetration into the seafloor and develop high resolution subsurface imagery. The subsurface imagery that is generated using sub-bottom profiling methods is scaled in a temporal domain (in milliseconds) vertically with respect to horizontal spatial domain (i.e., feet, or meters). Since, the speed of sound travelling through the media (water, silt, sand, etc.) is not constant during data acquisition, the accurate means of recording the data is by collecting the time it takes for signals to reflect from objects or strata and return back to the echosounder. Then it is possible to transform these time differences into distances using velocity models for the speed of sound in water, silt, sand, clay, etc. during processing and interpretation. The acoustic wave interaction with the seabed depends partially on interface between layers of different acoustic impedance (i.e. the product of sound velocity and density), part of the seismic energy reflects back to receiver, part of the energy is transmitted through the boundary to deeper layers and part of the energy is lost through scattering. The impedance contrast between the layers determines the amount of energy that is reflected back. The reflected energy is a function of relative densities, sound speed and angle of incidence at the two layers. The acoustic wave interaction at the seabed follows Snell's Law. SonarWiz5 data acquisition and post-processing software was used to collect sub-bottom data.[5]

\section{DATA COLLECTION METHODOLOGY:}

For data collection using the acoustic equipment above one should always be vigilant of the water depth the equipment is being used. Higher frequencies should be avoided in shallow-water unless required, but then should always monitor the system continuously to avoid overloading of the transmitter. The transmit power should be controlled in shallow areas as (i) the ping rate in shallow areas is very fast enough not allowing the transmitter to discharge fully; (ii) the reflected signals will be high in amplitude that will cause reverberation in transducer and may damage them, and (iii) the multiples of the signals will be picked up that will be mixed with the first reflections giving wrong data information. This is true in all acoustic equipments. An e.g. is shown in fig 1 and fig 2 Ship time is very precious, hence, before setting out for any measurements all the equipments should be checked and set at the port. Some equipments need settling time, warm up time, powering of such equipments should be pre-planned. Many a times positioning systems, do not deliver proper positions but is displayed on the instrument screen, and if not monitored continuously for some time one will consider the system is working, and will always record same wrong location values for the data. To avoid this, one should always check the positioning system in terminal mode for the desired string values (\$GPGGA, 
\$GPZDA) and their update in terms of time, and position.

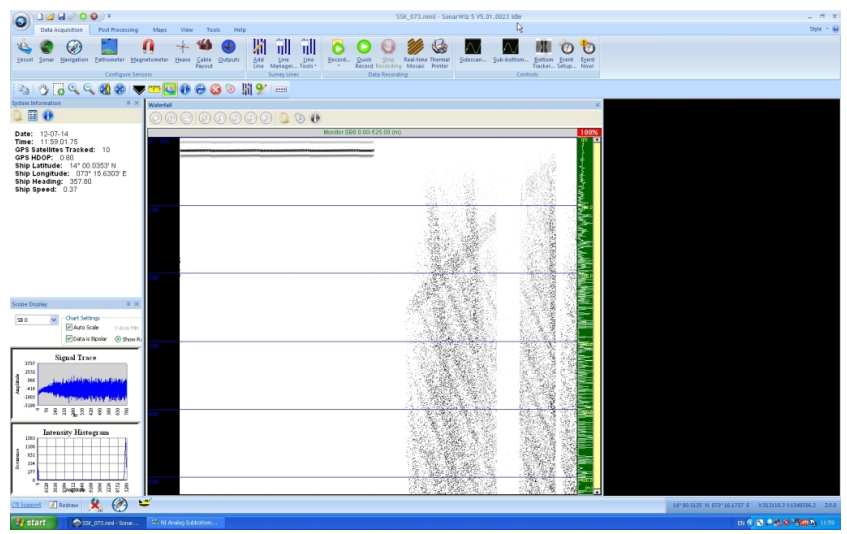

Fig. 1. Gain \& range parameters not set correctly, introduces large noise resulting in suppression of actual echo signal.

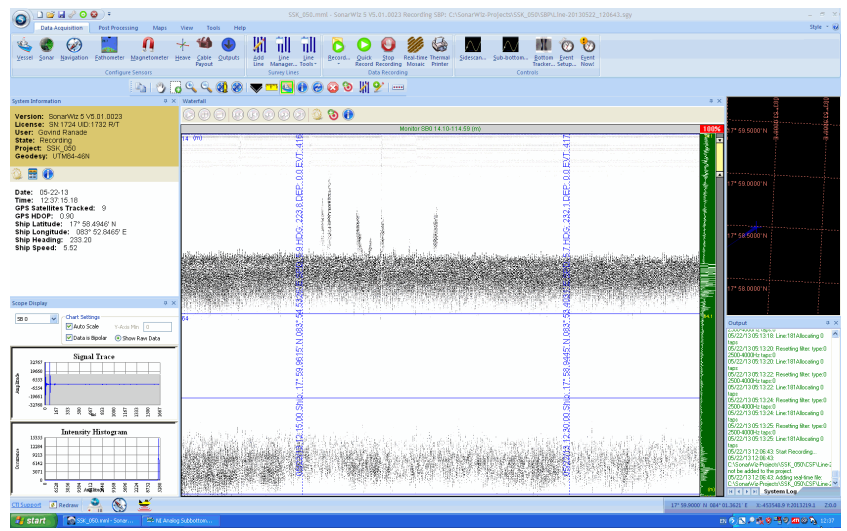

Fig. 2. Online data in shallow region with proper setting of gain, range and other parameters have resulted in a well defined bottom \& sub-bottom traces along with multiple echo.

On a steep slope region the echosounder usually looses the echo, and hence proper depth cannot be achieved. During data acquisition it is necessary to have good knowledge of the area being surveyed from the available charts. Now wherever there are steep slopes, one needs to be vigilant and set the gate limits i.e. min-max depth continuously, to get the good quality data over the slope. Similarly for Sub-bottom profiler, one needs to continuously monitor the amplitude window, and set the gain and power accordingly to avoid the overlapping of multiple signals onto the first echo.

As discussed earlier, in case of multibeam sonar, sound velocity profile and sound velocity near the transducer plays very important role in orientation of beams. Hence a close watch on the two values is essential. There are also other parameters that need to be looked for like the intensity window, cross track window and numerical window as shown in fig. 3 . The intensity window will give indication of quality of the beams. The cross track window should be monitored for the shape of the curve. When a slope is encountered, the cross track window needs to be continuously monitored and set to get the depth cross profile. Having seen a smiley shape could be an indication for large variation in sound velocity, and can be confirmed by checking the two values in numerical window. If there is a difference of more than $5 \mathrm{~m} / \mathrm{s}$, it is time to take new sound velocity profile in the area. Also temperature plays a major role in sound velocity; hence normally advised to have minimum three profiles in a day i.e. early morning, one in the noon and one in the evening. Sound velocity also depends on the salinity, hence while working in near coast area where rivers discharge into the sea, frequent profiles are required to compensate the salinity changes and the effects of tidal variation which is more prominent in near coastal zones.

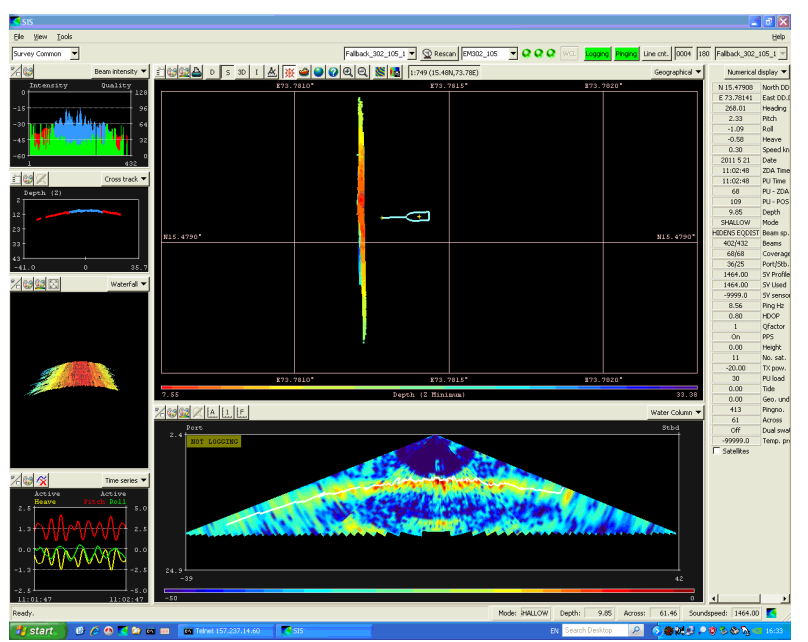

Fig. 3. Incorrect gridding parameters and absence of SVP data settings, result in improper gridding data display for monitoring purpose. Also absence of SVP introduces refraction errors as seen in cross track profile.

In fig 4 a proper data with proper compensation of the sound velocity in the vertical column and for the roll pitch and roll data is shown. These are gridded data with a grid cell spacing considered at $10 \%$ of the water.[6]

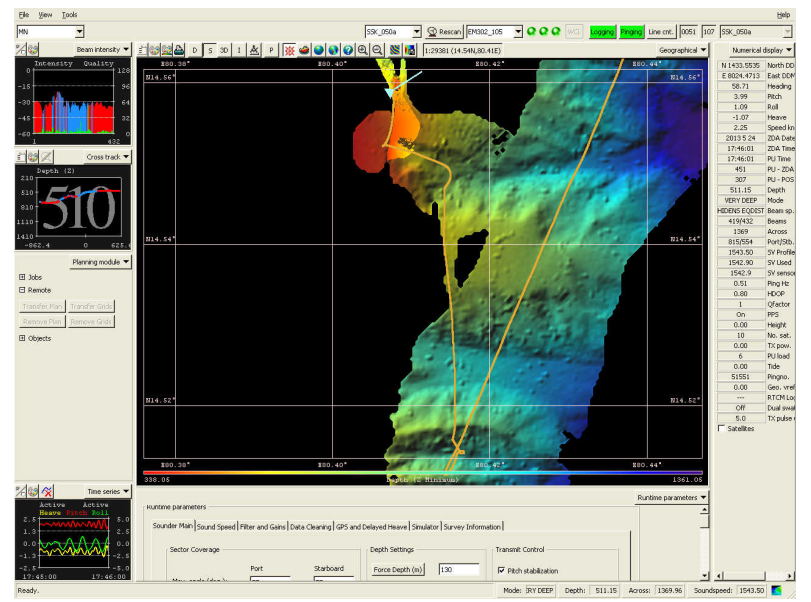

Fig. 4. On introducing proper parameters the data can be seen in proper perspective. 


\section{CONCLUSION:}

For acquiring a good quality data, it is very important that the systems are chosen according to the application and kind of the vessel on which they are being installed. It is also very important to carry out dimensional survey with adequate accuracy, so that very precise positional offsets of each of the sensor with respect to each other are achieved. It has been observed that a close monitoring on different parameters is required to achieve a good quality data. Calibration process is also an important aspect, which cannot be ignored. With the advent of laser ranging systems, it is possible to get precise installation parameters, which shall govern the calibration results. A good sound velocity data in the water column plays a major role in obtaining a good quality data. In future a continuous sound velocity system can provide a real time vertical sound velocity structure in the water column.

\section{ACKNOWLEDGMENT}

The authors $1 \& 2$ are thankful to Director, CSIR-NIO, for providing facilities to carry out this study. The authors 1 \& 3 would also like to thank Sh. Pushpashil Satardekar of Electronics and Telecommunication Engineering department at Goa College of Engineering, for his support and help.

\section{REFERENCES}

[1] Christian de MOUSTIER. State of the art in swath bathymetry survey systems. Internantional Hydrographic Review, 65(2):25-54, July 1988.

[2] R. Urick. Principles of Underwater Acoustics. McGraw Hill, 1975.

[3] Ranade Govind and Tata Sudhakar. Multibeam swath bathymetry signal processing techniques. In Proceedings of the National Symposium on Ocean Electronics, pages 71-75, 1991.

[4] Jeroen Dunnewold. Dynamic calibration of multibeam systems,. Technical report, Delft University Press, 1998.

[5] SonarWiz5 User Guide, Rev 5.03.0008.

[6] SIS Seafloor Information System reference manual 337660/E. 